\section{Detection of changes in spatial position: Short-term visual memory or motion perception?*}

\author{
IRWIN POLLACK† \\ University of Michigan, Ann Arbor, Michigan 48104
}

The limiting conditions for short-term visual memory were explored for materials which are not readily codable. Two fields of random dots were presented successively in time. On half of the observations, the second display was identical to the first, and on the other half, a fraction of the dots was displaced relative to the first display. The task of the $S$ was to indicate if any of the dots had moved (displacement detection), and in separate tests, if any of the dots had not moved (stationarity detection). Stationarity and displacement detection are largely related to the same variables, although performance is somewhat better with displacement detection. Discrimination of small displacements is critically dependent upon the interval between displays, with maximum accuracy in the region of $64 \mathrm{msec}$ for briefly presented displays. Maximum accuracy is obtained under conditions which yield good apparent motion. By contrast, displacement discrimination is, within limits, relatively independent of the number of dots displayed, of the fraction of displaced dots. of the brightness of the display, and, even of the magnitude of displacement. Is this a relatively crude visual short-term memory, unencumbered by coding factors, or simply the visual detection of motion?

The judgment of visual "sameness" seems-phenomonally, at least-to defy the step-by-step serial analysis often associated with selective visual tasks. Posner and Mitchell (1967), for example, report that the judgment of the same "name" of alphabetic characters is faster when the actual visual shape of successive letters was identical. Specifically, when $S$ s are instructed to respond to the same "name," faster response times are attained when $A$ is successively followed by $A$ than when $A$ is followed by a. The advantage of identical shape nearly disappears when the successive letters are separated by 1.5 to $2 \mathrm{sec}$ (Posner \& Keele, 1967; Posner et al, 1969). Presumably, the primitive representation of the first character either fades in the intermediate time period and/or is displaced by a "naming" or categorization response (Pollack, 1959). In any event, a short-term visual memory may be indicated. Such a short-term visual memory is also indicated in Sperling's (1960) work with alphanumeric characters and in subsequent efforts to explicate the

\footnotetext{
*Research supported in part by Grant G B 14036X of the National Science Foundation. The writer acknowledges the able assistance of Mrs. Nancy Mandell for supervising the experimental tests, of Lon Radin for the computer program, and of Robert Hsieh for processing the experimental results. He is also indebted to Professor Matthew Alpern for comments on the control experiment and to Dr. Charles Robinson for discussions in the formative stages of the research.

+Mailing address: Mental Health Research Institute, University of Michigan, $205 \mathrm{~N}$. Forest Street, Ann Arbor, Michigan 48104 .
}

visual "icon" (see Neisser, 1967, for a summary).

In a recent paper, Phillips and Baddeley (1971) criticize the logical implications drawn from the Posner experiments with respect to short-term visual memory. They argue that for codable items such as letters, the development of the name code overtakes the fading visual image. Although the visual image continues to fade, performance is governed by the name code. They first exposed 5 by 5 matrices of randomly filled squares for 0.5 sec, then a "noise field," and then a reproduction of the first field with a single square deleted or added. They observed increments in response time and in the number of errors, as the interval between the two fields was increased. Performance was above chance for intervals of 3 sec.

Sekuler and Adams (1968) explored the comparison between simultaneously presented random-cell matrices. While this study was not concerned with short-term visual memory, it provided provocative speculations of the nature of visual comparison processes. Specifically, their Ss viewed two 4 by 4 matrices, presented side by side, with both matrices consisting of 1,2 , or 4 filled cells. The number of cells in exact registration varied from zero to the number of filled cells. When not in registration, the filled cells were chosen at random. Two groups of Ss were employed: one judged whether or not "any" of the cells were in registration ("visual similarity"); the other judged whether or not "all" of the cells were in registration ("visual identity"). Response times were markedly faster in the latter case than in the former. Moreover, response times were relatively insensitive to a wide range of display variables in the latter case, in contrast to the former case. They conclude that, whereas response to visual similarity indicated "a serial processing of the stimulus array," the response to visual identity "closely approximated Gestalt processing," presumably referring to the extremely fast processing suggested by their data.

Kinchla and his associates (Kinchla, 1971 , and references cited therein) have studied absolute movement perception for a single point in an otherwise dark field and relative movement perception with a stable reference point in the visual field. With the aid of a signal detectability analysis, Kinchla has successfully parceled out criterion and discriminability factors and distinguishes memory noise from input noise. Memory noise, estimated from his observations, increases linearly with the interval between exposures.

The present tests examine the detection of displacements and of nondisplacements for successively presented random dot patterns. They aim to explore short-term visual emory for relatively uncodable displays.

\section{I, DETECTION OF DISPLACEMENT IN VISUAL POSITION Method}

Experimental variables and task definition. The memory for visual position was explored by presenting two random dot fields, separated by a time interval. The task of the $S$ was to indicate whether the position of the dots in the second field was identical to or different from the first. Specifically, a field of $\mathbf{n}$ randomly generated dots was exposed for $t_{1}$ msec; after an interflash interval, or IFI, a second field of dots was exposed for $t_{2}$ msec On one-half of the

\begin{tabular}{ll}
\hline Number of moved dots in the second flash, displaced relative to the first test flash \\
$n$ & Number of dots in each test flash \\
$t_{1}$ & Duration of the first test flash \\
$t_{2}$ & Duration of the second test flash \\
IFI & Duration between the two test flashes \\
d & Displacement of dots between the two test flashes \\
$D$ & Distance of observer to the display \\
$s$ & Number of stationary dots in the second flash, not displaced relative to the first test flash
\end{tabular}


Table 2

Comparison of Coherent (I) and Incoherent (II) Shifts for Displacement (D) and Stationarity (S) Detection

\begin{tabular}{|c|c|c|c|c|}
\hline & \multicolumn{2}{|c|}{ A. Displacement Detection } & \multicolumn{2}{|c|}{ B. Stationarity Detection } \\
\hline & I > II & II $>$ I & $\mathbf{I} \cdot \mathbf{S}>\mathbf{I I}-\mathbf{S}$ & II-S $>$ I-S \\
\hline Direction & 176 & 319 & 66 & 33 \\
\hline$p<.05$ & 32 & 70 & 38 & 10 \\
\hline $\mathrm{p}<.02$ & 20 & 49 & 33 & 4 \\
\hline $\mathrm{p}<.01$ & 11 & 34 & 29 & $\mathbf{3}$ \\
\hline
\end{tabular}

C. Average Scores (Percent)

\begin{tabular}{llll}
\hline II-D & $\mathbf{8 3 . 0}$ & D & $\mathbf{8 1 . 7}$ \\
I-D & 80.4 & S & 73.7 \\
I-S & 76.0 & II & 78.2 \\
II-S & $\mathbf{7 1 . 4}$ & I & 77.2 \\
\hline
\end{tabular}

\begin{tabular}{lccccrr}
\hline & & D. Direction & & \multicolumn{2}{c}{ E. Intercorrelation } \\
& I & I-S & II-S & I & I-S & II-S \\
\hline II & 71 & 85 & 95 & .91 & .82 & .77 \\
I & & 84 & 82 & & .92 & .72 \\
I-S & & & 66 & .76 \\
\hline
\end{tabular}

observations, the second field was identical to the first. A correct response upon these trials was to press the key marked "same." On one-half of the observations, $(n-m)$ of the dots of the second field were identical to the first, whereas $m$ randomly selected dots were displaced by $\mathrm{d} \mathrm{mm}$. A correct response on these trials was to press the key marked "moving or displaced." Table 1, except for the last line, defines the variables for the detection of visual displacement.

A PDP-8 computer generated random dot fields upon a 1024 by 1024 matrix to encompass an $8 \times 8 \mathrm{~cm}$ area of a Tektronix 602 display scope (about $2.2 \mathrm{deg}$ of visual angle). A low-level background luminance, about $0.01 \mathrm{~mL}$, and a fast P15 phosphor served to minimize display persistence. Unleśs noted otherwise, $S$ sat $210 \mathrm{~cm}$ from the scope. Natural binocular viewing was employed. A random field of 100 dots had a luminance of about $10 \mathrm{~mL}$, as measured by a SEI photometer. All dots in a display field were plotted successively at the maximum plotting rate of the computer program (24 microsec/point), and the entire display was successively refreshed to achieve the exposure duration, $t$. The brightness of each dot was determined primarily by the number of paintings and/or the display duration. For any one dot of a display, its display duration was approximately $t / n$. The position of each dot was selected randomly, with a uniform distribution over the display, with the following restriction: an imaginary square, $2.5 \mathrm{~mm}$ on a side, was positioned about each random dot selection; if a later dot selection violated that area, whether upon the first or upon the second display, that selection was dropped and another random selection was drawn.

Procedure. Two series of tests were run: in visual memory for Position I (VMP I), displacements of the $m$ dots of the second field were all $x$ displacements to the left; in visual memory for Position II (VMP II), the displacement of each of the $m$ dots in the second field was randomly selected from four directions: northeast, southeast, southwest, and northwest. A displacement of $d$ units in VMP I is translated into a resultant displacement of $d \cdot(2)^{1 / 2}$ in VMP II, since the displacement of $d$ units was impressed upon both the $x^{-}$and $y$-coordinates. Sixteen observations constituted a trial. Each score was the number correct out of 16 observations. Immediate feedback was given after each response. No attempt was made to distinguish between misses and false alarms (cf. Kinchla, 1971 ), since the $S$ could modify his criterion for "sameness" with feedback. The minimum response-display interval was $1.5 \mathrm{sec}$ for displays with a small number of dots. Calculation of displays with 100 dots required up to 16 sec. A rough idea of the number of calculations required for displays with a large number of dots is obtained by reference to the 1.5 microsec cycle time of the computer.

Fourteen Ss, with previous intensive experience with both auditory and visual displays, together contributed 25-36 scores (median, 28; mean, 30) for each of the experimental conditions under VMP I and the same number under VMP II. Thus, each average score is based upon about 480 observations. When results were averaged under VMP I and VMP II, 960 observations. There were 495 experimental conditions under both VMP I and VMP II. With additional control tests, this resulted in about $0.6 \times 10^{6}$ observations. Unless noted otherwise: $\quad m=4, \quad n=8$, each condition is based upon about $t_{1}=t_{2}=12 \mathrm{msec} ;$ IFI $=64 \mathrm{msec}$, $\mathrm{d}=2.5 \mathrm{~mm}, \quad \mathrm{D}=210 \mathrm{~cm}$, and the plotting area was $8 \times 8 \mathrm{~cm}$. At the viewing distance of $210 \mathrm{~cm}$, the plotting area subtended a visual angle of about $2.2 \mathrm{deg}$; the equivalent angular displacement for $\mathrm{d}=\mathbf{2 . 5} \mathrm{mm}$ was about $4 \mathrm{~min}$.

\section{Results and Discussion}

Comparison between VMPI and VMPII. The product-moment correlation between the percent correct scores, calculated for 396 of the experimental conditions, was 0.913 . The average ratio of scores on VMP I, relative to VMP II, was 0.997 . Whatever advantage accrued by correlated movement in VMP I apparently was nearly exactly compensated by the resulting $(2)^{1 / 2}$ greater displacement in VMP II. The first conclusion, then, is that when examined over a wide range of conditions, the memory for visual position is not appreciably different for coherent displacement than for a more random displacement. This conclusion is qualified in Table 2-A, which lists the number of experimental conditions (out of 495) which demonstrated a difference in sign and a significant difference score (uncorrelated $t$ test) at confidence levels of $5 \%, 2 \%$, and $1 \%$ in favor of VMP I and in favor of VMP II. The largest apparent difference between VMP II and I obtains at the highest confidence level. This apparent superiority of VMP II must be interpreted with caution: although most of the Ss were common to both series, a fraction of Ss contributed scores primarily to only one of the series.

Discrimination of displacement as a function of the number of dots displaced. Is memory of visual position directly related to the fraction of displaced dots? An affirmative answer is expected if Ss attend to only a small fraction of the display. The probability of sampling a displaced dot will then be directly related to the fraction of displaced dots. A negative answer is expected, either if Ss can attend to the entire display or if the discrimination of displacement is enhanced by reference to nondisplaced dots. When all dots are displaced in the same direction in VMP I, for example, Ss may find it difficult to discriminate between displacements related to shift of eye position from experimentally introduced displacements.

Figure 1 presents discrimination scores for briefly flashed displays with an interflash interval of $64 \mathrm{msec}$. The abscissa of the left and middle sections is the total number of dots in the display for VMP I and for VMP II, respectively; the abscissa of the right 


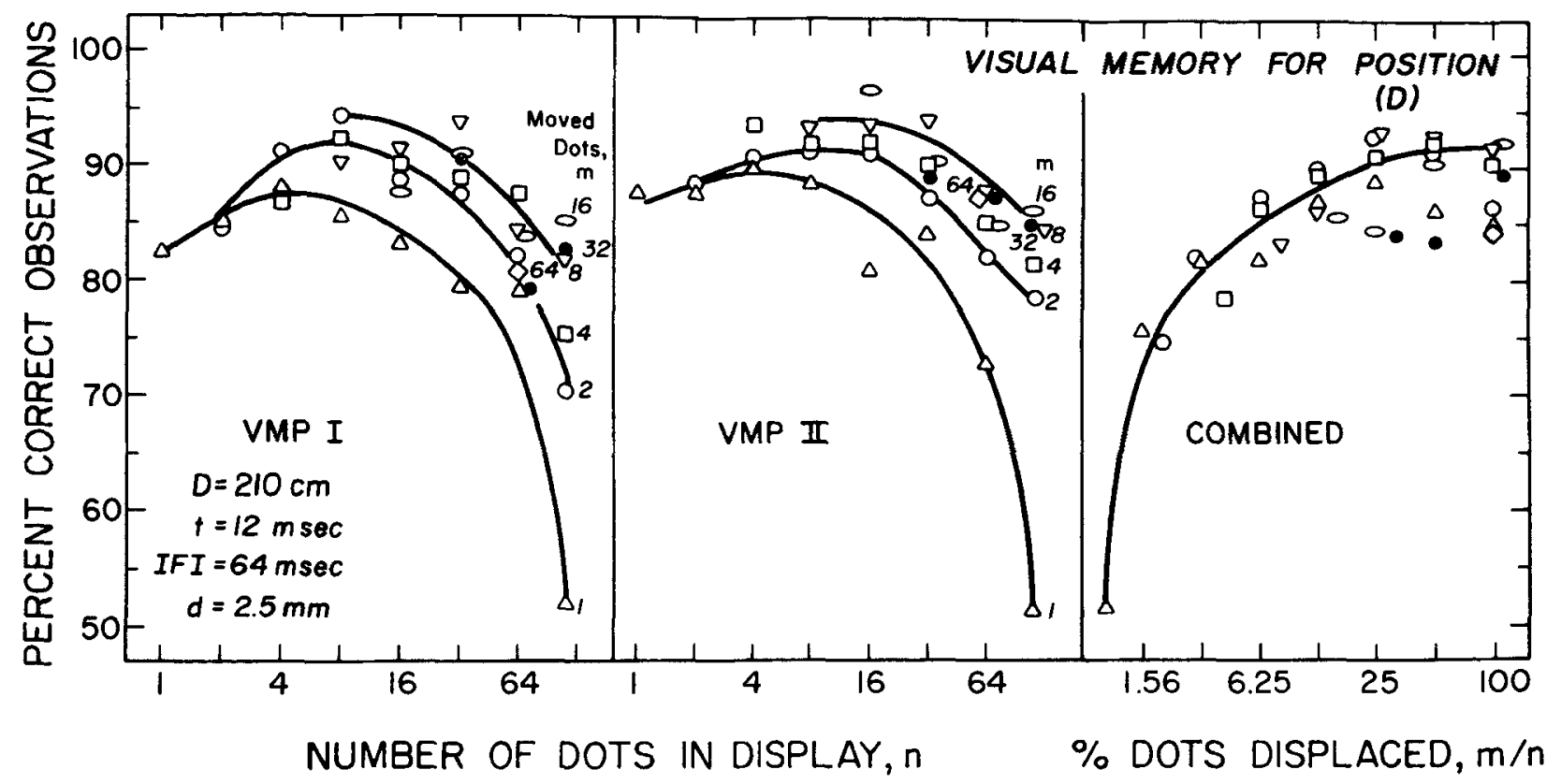

Fig. 1. Displacement detection as a joint function of the number of displaced dots (parameter) and of the total number of dots in the display (abscissa). The results for coherent displacement (VMP I) are presented in the left section; those for incoherent displacement (VMP II) are presented in the middle section. The right section combines the results for coherent and incoherent displacement as a function of the percentage of dots displaced.

section is the fraction of displaced dots, $m / n$. The parameter is $m$, the number of displaced dots. Results are pooled over VMP I and VMP II in the right section.

With just a single displaced dot, $m=1$ in the left and middle sections of Fig. 1., discrimination scores decrease with the number of dots of the display. As the number of displaced dots is increased, discrimination becomes nearly independent of the number of dots of the display. Alternatively, the right section suggests that discrimination is impaired primarily only with an extremely small fraction of displaced dots and is, thereafter, relatively independent of the number or fraction of displaced dots. The relative independence of performance upon the number of dots in the display might suggest that Ss were effectively sampling only a section of the entire display. The outstanding difficulty with this sampling hypothesis is that performance is nearly maximal when as few as $1 / 16$ of dots were displaced.

Finally, the right section hints that discrimination accuracy may actually decrease when the fraction of displaced dots is increased from 50\% to $100 \%$, as might be expected if displacement discrimination is aided by reference to nondisplaced items (cf. the distinction of Kinchla, 1971, between absolute and relative movement discrimination).

Discrimination of displacement as a function of distance to display. Four different display displacements were also run at each of four distances to the display. The left section of Fig. 2 considers discrimination scores as a function of the display displacement. The parameter is the distance to the display. If discrimination were determined by retinal displacement alone, the points would be spread horizontally, as shown in the scaled insert. Scores are lower for the longer display-observer distances, but the spread is substantially smaller than that expected on the basis of retinal displacement alone. Except for extremely small retinal displacements, discrimination appears to be more closely tied to display, rather than to retinal, displacement.

In additional tests, 1,2 , or 4 dots were displaced within a display of 64 dots. The distance of the $S$ to the display was varied. The right section of Fig. 2 considers discrimination scores as a function of distance to the display (abscissa) as a function of the number

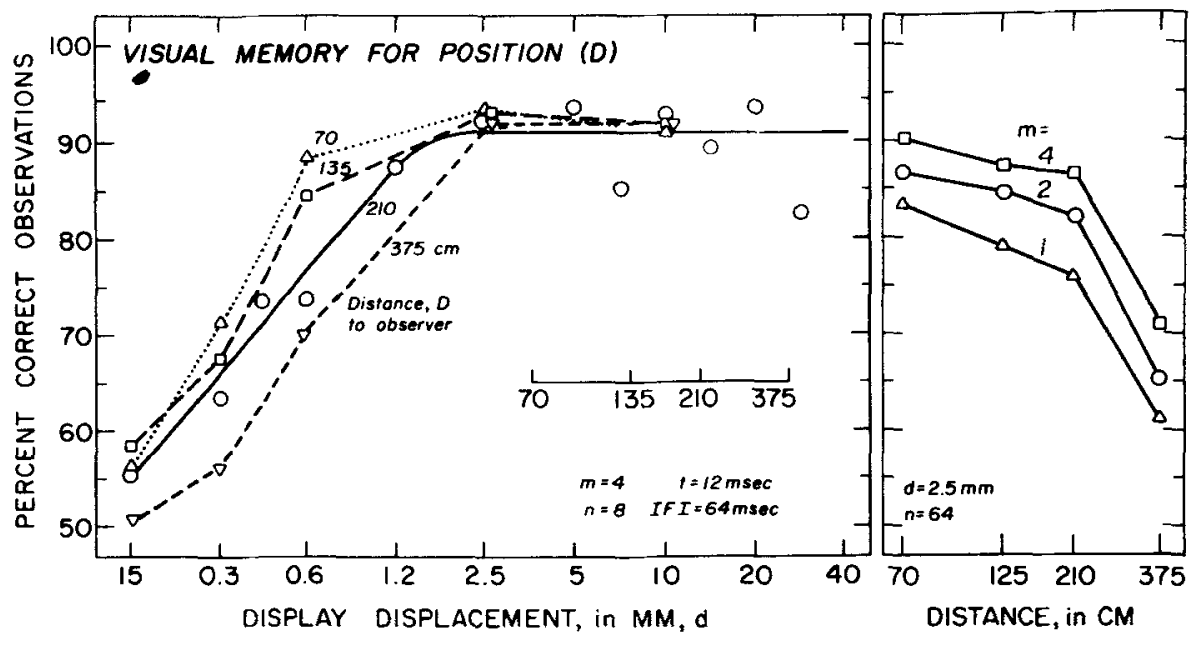

Fig. 2. Displacement detection (left section) as a function of the magnitude of the displacement of dots within the second display and of the distance between the $S$ and the display (parameter). Displacement detection (right section) for displays with a small number of displaced dots (parameter) as a function of display distance (abscissa). 


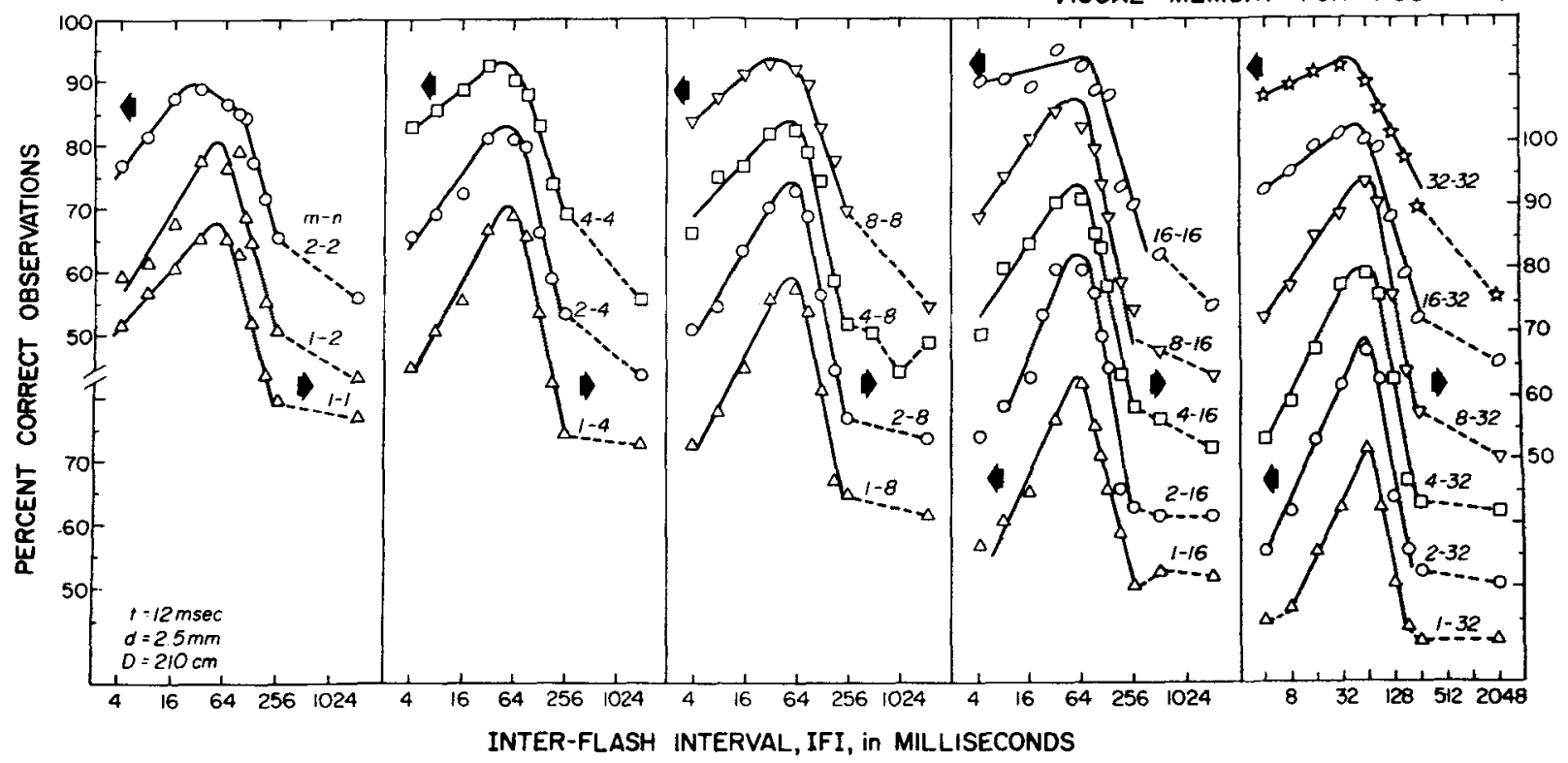

Fig. 3. Displacement detection as a function of the interval between the first and second displays (abscissa) for brief displays. The parameter on the curves reflects the number of dots moved or displaced and the total number of dots in the display. Within each panel, successive functions are displaced downward by 10\%. For example, the leftmost point associated with 1-2 (left section) is plotted at $59.1 \%$ correct, relative to the scale associated with the top $2-2$ curve, and performance should be read as $69.1 \%$ correct. Similarly, the rightmost point associated with $4-32$ (right section) is plotted at $42 \%$ correct, relative to the scale on the right associated with $8-32$, and should be read as $52 \%$ correct. This method of plotting is employed in Figs. 3, 4, 5, and 8-15.

of displaced dots (parameter). Apparently, it is somewhat easier to detect the movement of a small fraction of dots when the display field occupies a larger retinal area than when it occupies a smaller retinal area, at least for the conditions of testing of the right section of Fig. 2.

Discrimination of displacement as a function of the interflash interval, IFI. Figure 3 considers discrimination scores for those conditions within Fig. 1 which were examined over a range of interflash intervals. To aid the graphic presentation, successive curves are displaced by $10 \%$. The parameter on the curves is the number of displaced dots and the number of dots in the display. Figure 3 suggests that, for the moderate displacement of $2.5 \mathrm{~mm}$ (4 min of visual angle) of brief (12-msec) flashes, there is a relatively sharp maximum score in the vicinity of an IFI of $64 \mathrm{msec}$. Discrimination accuracy falls both at longer and at shorter IFIs. Phenomenally, the Ss report that displacement changes in the region of $64 \mathrm{msec}$ are associated with perceived movement, changes at low IFIs are associated with perceived number or brightness, and changes at high IFIs are associated with a cognitive change in apparent position. The relative invariance of the maximum of the discrimination functions with changes in $m$ and $n$ is striking.
Discrimination of displacement as a function of the magnitude of displacement of visual position. The accuracy of discrimination of a change in visual position is obviously related to the magnitude of the change. The unfilled circles and the heavy curve of the left section of Fig. 2, however, suggest that this relation is not direct. Small displacements are hardly detectable, but extremely large displacements are scarcely more discriminable than are intermediate displacements. The apparent ceiling with large displacements may have been due to the difficulty of achieving good perceived motion with extremely large displacements, to $\mathrm{S}$ inattention on some fraction of trials, or it may have resulted from Ss' responding "different" when expecting small displacements in the first several observations. The experimental data do not discriminate among these possibilities.

Figure 4 examines the role of interflash interval for displacements of different magnitude. As in Fig. 3 , the ordinate has been displaced for successive conditions tested. At least for the conditions of testing examined, the peak in performance in the vicinity of IFI $=64 \mathrm{msec}$ is obtained over all displacement magnitudes. While the empirical functions broaden for larger displacements, there is no hint that the peak of the functions shifts with displacement magnitude in order to maintain a constant displacement velocity.

Discrimination of displacement as a function of display duration. In the previous tests, the duration of the first and second test flashes was short $\left(t_{1}=t_{2}=12 \mathrm{msec}\right)$. Are the strong iffects of IFI obtained in Figs. 3 and 4 pecific to briefly presented displays? erhaps.

Figure 5 examines the role of interflash interval for a range of display durations for the case where $t_{1} \stackrel{=}{=} t_{2}$. . As in Figs. 3 and 4 , the ordinates of successive curves are displaced by $10 \%$ to aid graphic presentation. For longer display durations, performance is best at shortest interflash intervals and decreases slowly until an interflash interval of about $64 \mathrm{msec}$ is achieved. As the display duration is made briefer, the plateau at the shorter interflash intervals gives way to the sharp peak in performance at intermediate IFIs, as in Figs. 3 and 4. Nor is the result specific to the displacement magnitude of $2.5 \mathrm{~mm}$. Figure 6 extends the inspection of the interaction between display duration and IF I to three displacement magnitudes (represented in the three sections). The transition from a plateau at short IFIs to a peaked function as the display duration is decreased is observed for each 


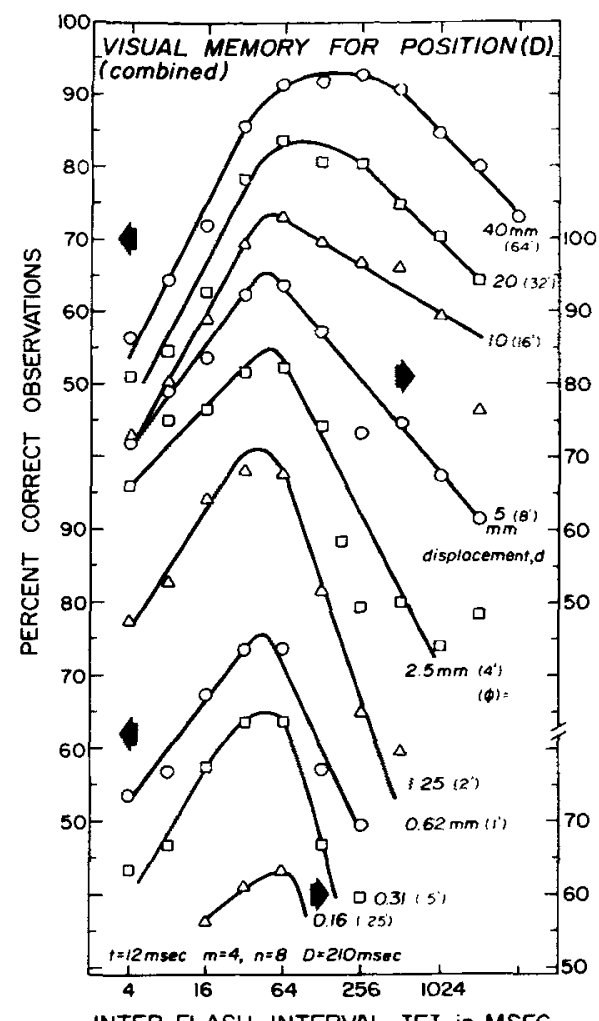

INTER-FLASH INTERVAL, IFI, in MSEC

Fig. 4. Displacement detection as a function of the interval between successive displays (abscissa) and the magnitude of displacement (parameter). The magnitude of displacement is expressed in terms of the horizontal component of the displacement in linear terms and in terms of the equivalent angular displacement).

displacement magnitude.

\section{DISCRIMINATION OF NONDISPLACEMENT OF VISUAL POSITION}

Following Sekuler and Adams (1968), separate tests were carried out in which Ss indicated whether or not any of the dots of the second field were not displaced, relative to the first field.

\section{Method}

The variables are identical to the discrimination of displacement of visual position, except that the first variable of Table 1 is replaced by the last variable of Table 1 . On one-half of the observations, all $n$ dots were displaced; on one-half of the observations, $(n-s)$ dots were displaced and $s$ dots were not displaced, relative to the first field. A correct response on the latter observations was to press the key marked "some stationary;" a correct response on the former observations was to press the key marked "none stationary." A subset of 99 experimental conditions representing some of the major points of Figs. 1-6 was selected for study. Again, each condition is represented by about 30 blocks of 16 trials contributed by 14-16 experienced experimental Ss. With control tests, about $0.2 \times 10^{6}$ observations resulted.

Comparison between VMP I S and VMP II-S. As with the discrimination of displacement, the discrimination of nondisplacement is reasonably comparable for coherent (VMP I-S) and noncoherent (VMP II-S) displacement. Over 99 experimental conditions, the product-moment correlation was only 0.76 . However, the average ratio of scores on VMP I-S, relative to VMP II-S, was 1.065 , which is considerably larger than that obtained for displacement discrimination. Table 2-B lists the number of conditions (out of 99) which demonstrated a difference in sign and a significant difference score at confidence levels of $5 \%, 2 \%$, and $1 \%$ in favor of VMP I-S and in favor of VMP II-S. Note the reversal between coherent and incoherent displacement for stationarity detection, in contrast with displacement detection.

Comparison between discrimination of displacement and discrimination of nondisplacement. There is a moderate loss in performance in the discrimination of nondisplacement relative to the discrimination of displacement. Table 2-C lists the average percentage correct observations for displacement (D) and stationarity (S) detection, for coherent (I) and for incoherent (II) shifts for a

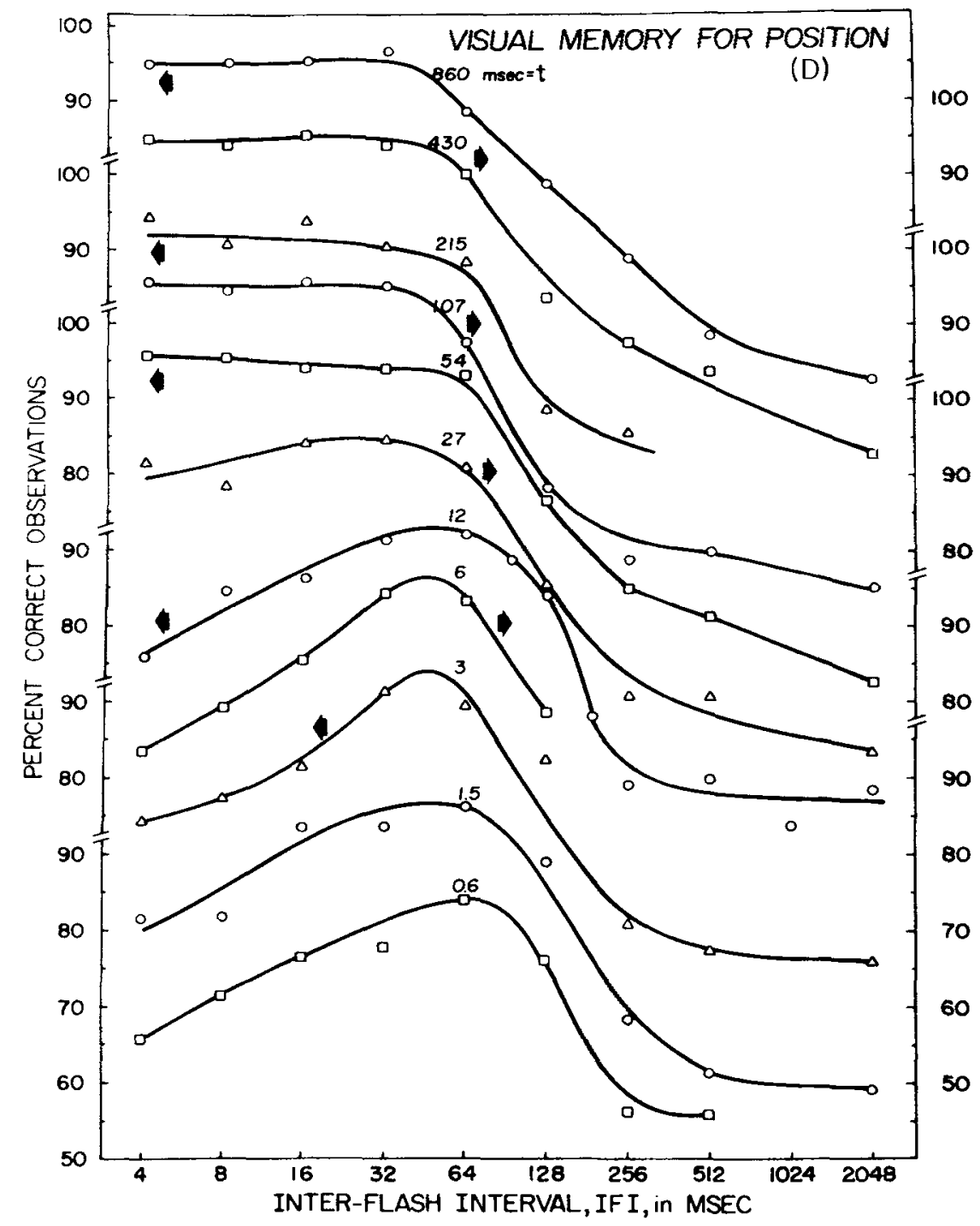

Fig. 5. Displacement detection as a joint function of interflash interval (abscissa) and display duration (parameter). 


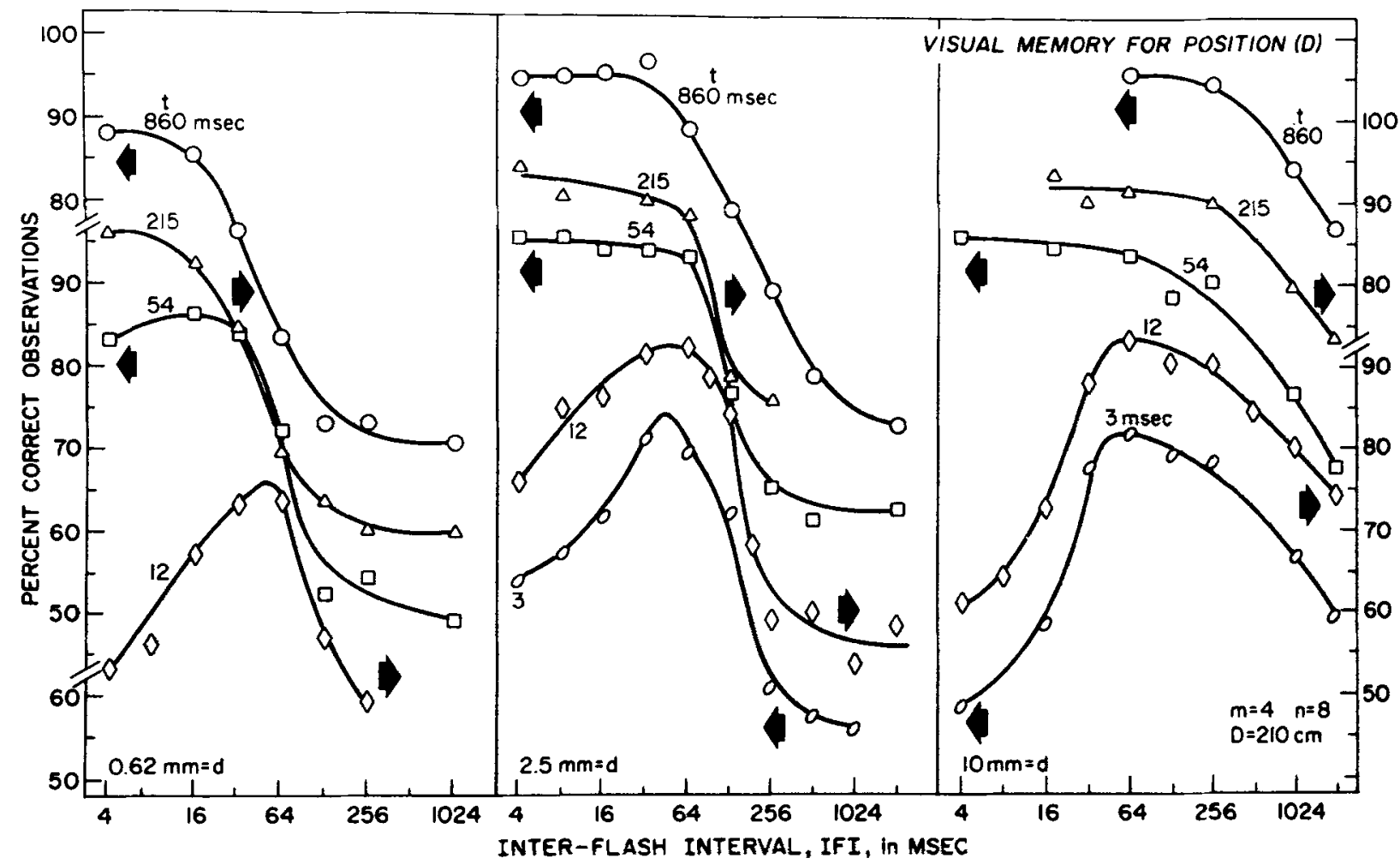

Fig. 6. Displacement detection as a joint function of interflash interval (abscissa), display duration (parameter), and magnitude of displacement (section).

common pool of 99 experimental conditions. For both forms of shifts, displacement detection is superior to stationarity detection.

Phenomenally, movement detection appears more compelling. The Ss also described their tasks in terms of the degree of movement: in displacement detection, their task was to detect the absence or presence of movement; in stationarity detection, their task was to detect the movement of all points from the movement of some points. The ethological literature abounds with descriptions of the survival importance of movement. There are also hints in the neurophysiological literature of the primacy of movement detectors (Lettvin et al, 1959).

With respect to the type of shift, there appears an interesting interaction: coherent shifts yield superior scores for stationarity detection; noncoherent shifts yield superior scores for displacement detection. Table 2-D presents the number of experimental conditions, out of 99 , in which the performance scores on the row method exceeded those on column method. Table 2-D supports the rank ordering within Table 2-C. Table 2-E considers the intercorrelation among the four methods, again based upon the common pool of 99 experimental conditions. The correlation in
Table 2-E between VMP I and VMP II is substantially lower than that previously obtained for a wider range of experimental conditions.

Discrimination of nondisplacement as a function of the number of dots not displaced. The organization of Fig. 7 parallels that of Fig. 1, except that Fig. 7 considers the number of nondisplaced dots. Over a wide range of conditions, discrimination of nondisplacement within Fig. 7 is substantially more sensitive to the fraction of nondisplaced dots than is the discrimination of displacement within Fig. 1. Consistent differences are found in favor of coherent displacement for stationarity

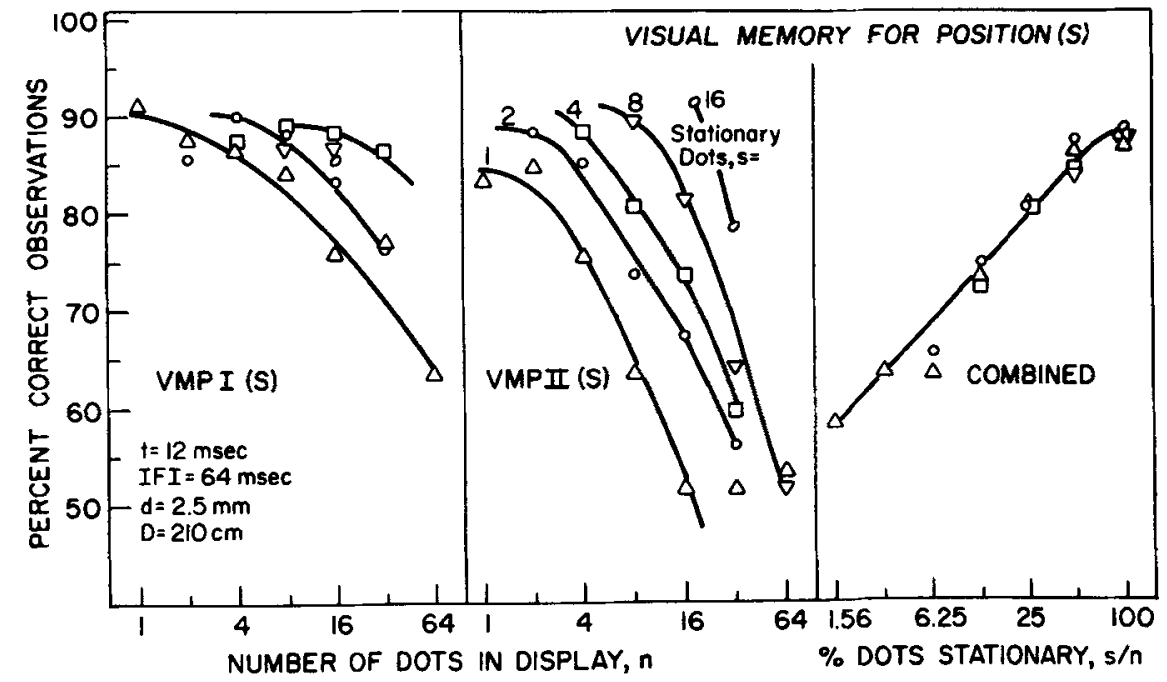

Fig. 7. Stationarity detection as a joint function of the number of nondisplaced dots (parameter) and of the number of dots in the display (abscissa). The organization is parallel to that of Fig. 1. 


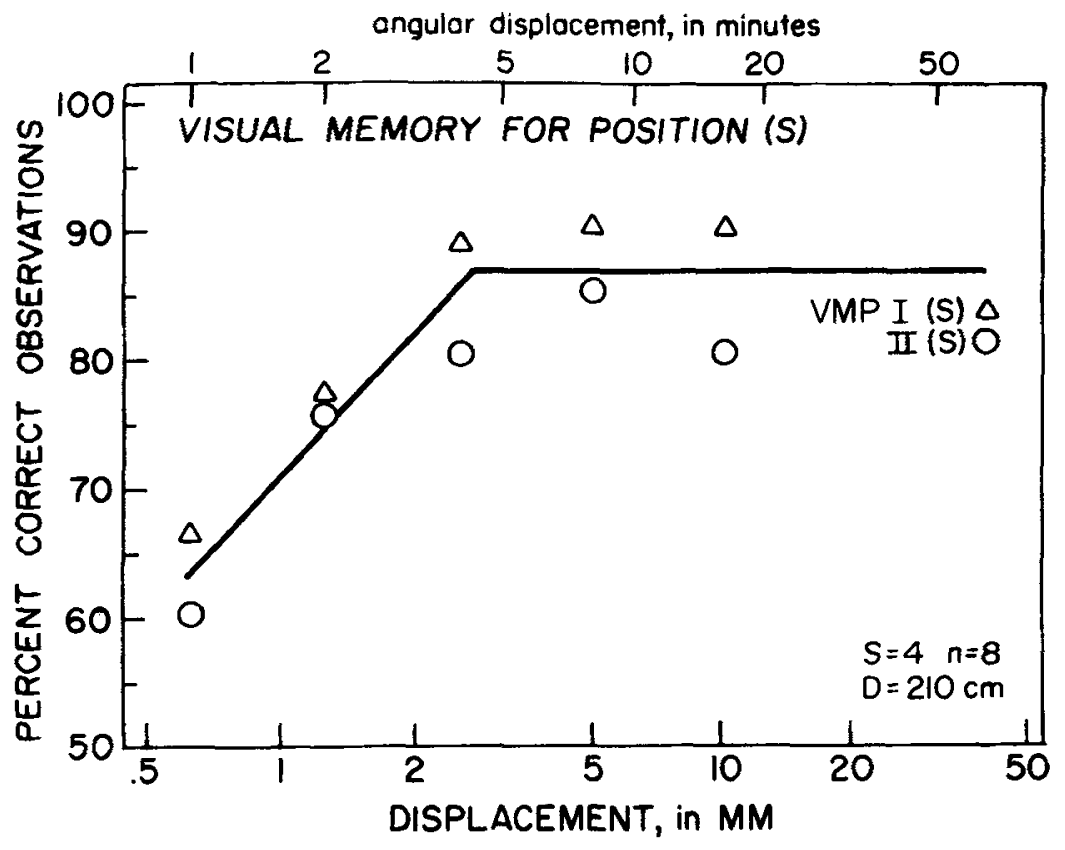

Fig. 8. Stationarity detection as a function of the magnitude of displacement of the displaced dots. The organization is parallel to that of the left section of Fig. 2.

discrimination, in contrast with the displacement discrimination tests of Fig. 1.

Discrimination of nondisplacement as a function of the magnitude of displacement. Under the conditions of the unfilled circles and heavy curve of the left section of Fig. 2, the tests for stationarity discrimination yielded the results shown in Fig. 8 . As in Fig. 2, there is a substantial improvement in performance for increments in the magnitude of small displacements. Again, as in Fig. 2, there is little further gain in performance beyond intermediate displacements.

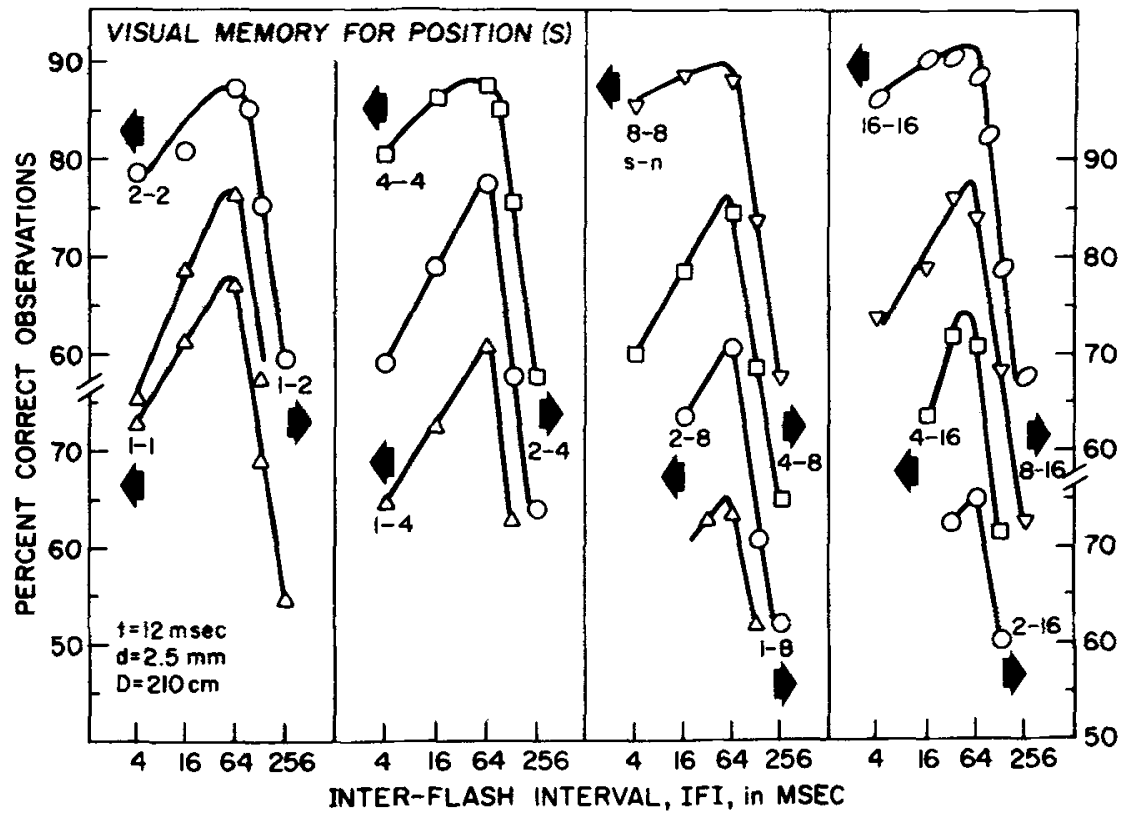

Fig. 9. Stationarity detection as a function of the interval between the first and second displays (abscissa) and of the number of nondisplaced and total dots (parameter). The organization is parallel to that of Fig. 3.
Discrimination of nondisplacement as a function of the interflash interval, IFI. The organization of Fig. 9 parallels that of Fig. 3. In Fig. 9, the number of nondisplaced dots is the parameter. As with the discrimination of displacement in the tests of Fig. 3 , the discrimination on nondisplacement in the tests of Fig. 9 is extremely sensitive to the interflash interval. Maximum performance is again achieved for IFIs in the vicinity of 64 msec for brief flashes. It is difficult to compare the sharpness of the maxima because fewer intermediate IFIs were examined in the tests of Fig. 9 than in those of Fig. 3 , and there is a difference in the absolute levels of performance. Over corresponding conditions, the average difference in the percent correct scores between IFIs of 4,16 , and $128 \mathrm{msec}$, below the corresponding scores at an IFI of $64 \mathrm{msec}$, is: $8.0 \%, 2.2 \%$, and $12.0 \%$, respectively, for displacement discrimination, and $6.1 \%, 1.2 \%$, and $17.4 \%$, respectively, for nondisplacement discrimination. On this basis, the maxima are somewhat sharper for the discrimination of nondisplacement, despite the lower performance levels at $64 \mathrm{msec}$ IFI for the nondisplacement tests $(89.8 \%$ vs 84.3\% correct).

Discrimination of nondisplacement and display duration. The organization of Fig. 10 parallels that of Fig. 5. The discrimination of nondisplacement in the tests of Fig. 10 is extremely sensitive to the interflash interval for briefly presented displays, but not for longer displays. This result closely parallels that of Fig. 5 .

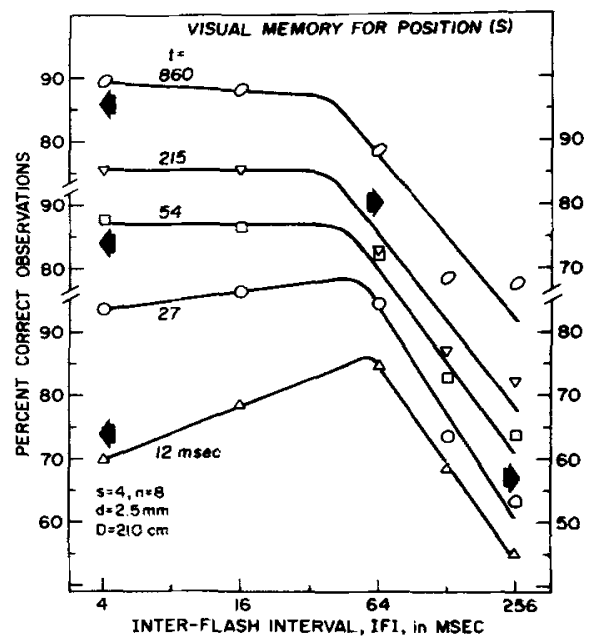

Fig. 10. Stationarity detection as a function of the interval between the first and second displays (abscissa) and of display duration (parameter). The organization is parallel to that of each section of Fig. 5. 


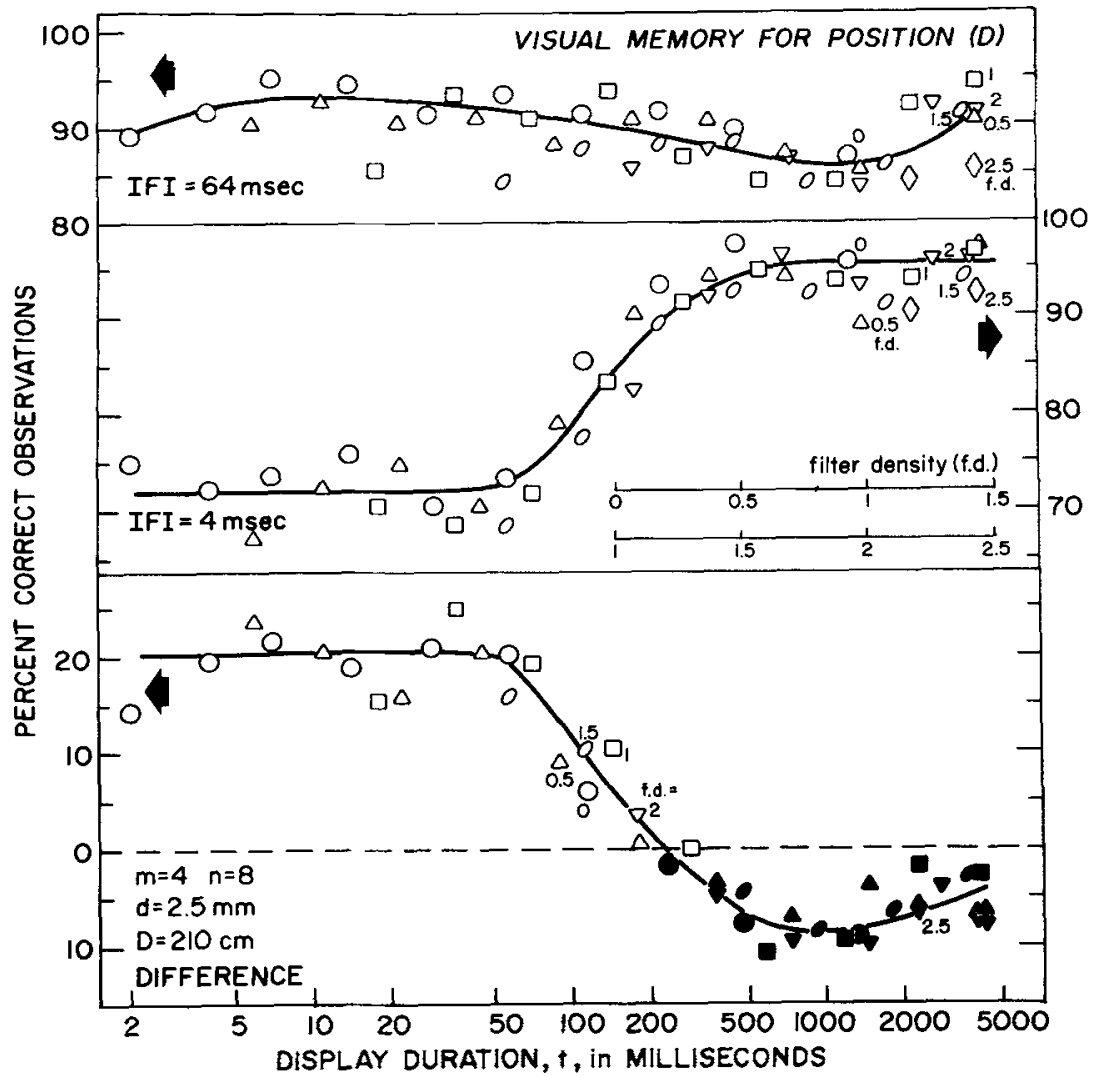

Fig. 11. Displacement detection as a function of display duration (parameter), filter density (parameter), and the interval between the first and second displays $(64 \mathrm{msec}$ in top section, $4 \mathrm{msec}$ in middle section, and the difference between the respective scores in the bottom section). Filled points represent difference scores below zero. The insert of the middle section is the expected separation of points if the product of filter transmission and duration were constant. Incoherent variation.

anticipate that the visual system integrates brightness for intervals to $100 \mathrm{msec}$, at least, and under the present low light levels, to even longer durations. The problem is to separate temporal factors unencumbered by brightness factors from brightness factors unencumbered by temporal factors. We cannot do this directly, but we have employed a series of experimental operations which attempt to tease apart their separate contributions.

Brightness vs Time

Consider two extreme hypotheses. The time hypothesis states that the change in shape of the IFI functions in

\section{DISCUSSION}

The most striking result from the extended testing program is related to the sharp maximum in detection performance for interflash intervals of about $64 \mathrm{msec}$ for brief displays. The fast drop-off in performance for IFIs above $64 \mathrm{msec}$ is presumably related to the fading of the memory trace of the display. This presumption is based upon the following observations: (1) performance is nearly at chance levels for IFIs of $250 \mathrm{msec}$, which has been taken as an estimate of the duration of the visual image; (2) detection accuracy associated with the rising portion of the IFI function can be manipulated by changing the proportion of displaced vs nondisplaced dots or by changing the display duration; detection associated with the falling portio of the IFI function appears to be more insensitive to such variations; (3) the fast drop-off, however, can be ameliorated by introducing large displacements, as might be expected if the precision requirements upon information in the fading image were substantially reduced.

The rising portion of the IFI function appears the more interesting because of its interactions with experimental conditions. We might

Fig. 12. Stationarity detection under the conditions of Fig. 11 .

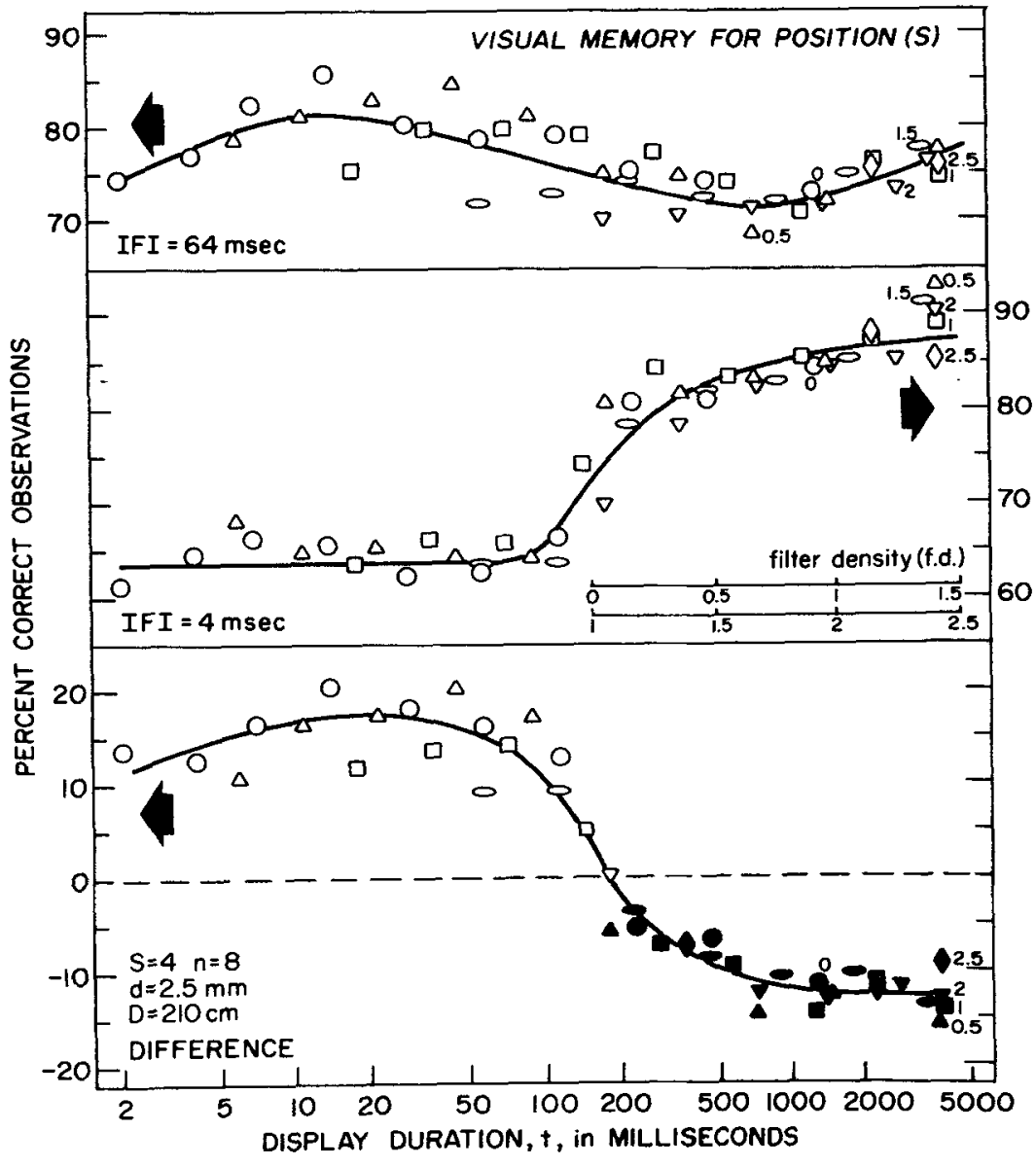

Perception \& Psychophysics, 1972, Vol. 11 (1A) 


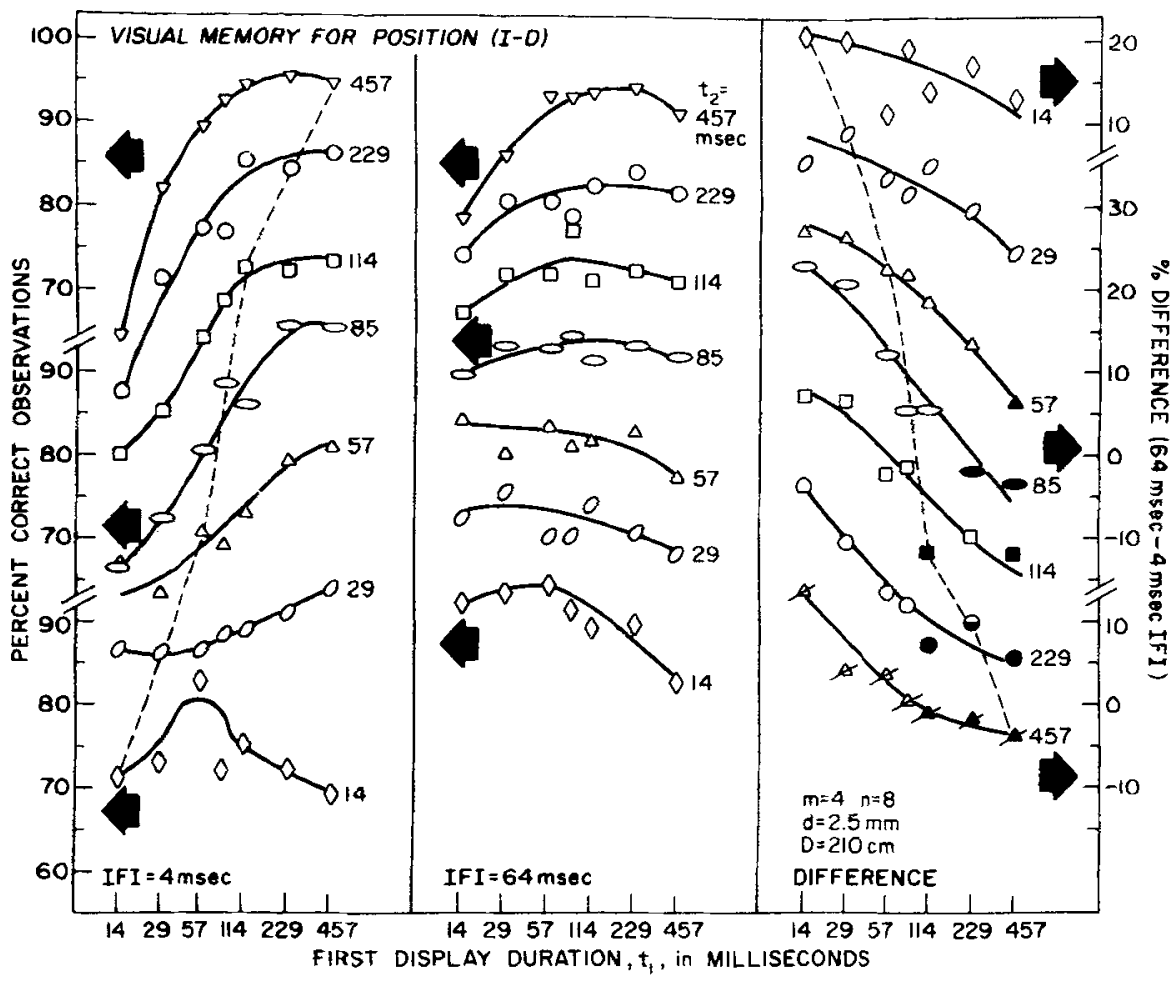

Fig. 13. Displacement detection as a function of the duration of the first display (abscissa), of the second display (parameter) for the indicated interdisplay intervals (left and middle sections). The difference scores are plotted in the right sections. Incoherent variation. The dashed lines connect points where $t_{1}=t_{2}$. top two sections of Figs. 11 and 12 represent performances obtained with $I F I=64 \mathrm{msec}$ and $\mathrm{IFI}=4 \mathrm{msec}$, respectively. The abscissa is display duration, and the shape of the points represent filter density. The lowest section represents the difference in performance under the two IFIs. The difference score fails to take into account the nonuniform sensitivity of the percent correct measure, but it does provide a simple measure of differences in performance. The insert in the middle section represents the expected displacement of points associated with different filters on the assumption of reciprocity between intensity and time. This reciprocity may be unreasonable for extremely long durations but is reasonable for the shorter display durations. In terms of Figs. 11 and 12 , the time hypothesis predicts that the points representing the different filters will fall on top of each other, i.e., performance will be determined by display duration alone. In terms of Figs. 11 and 12, the brightness hypothesis predicts that the points representing the different filters will be spread out in the manner shown in the insert, i,e, performance will be determined by the effective brightness alone.

The results of Figs. 11 and 12 clearly support the time, relative to brightness, hypothesis.
Figs, 5 and 10 with display duration is due to the duration of the display, irrespective of its brightness. The brightness hypothesis states that the corresponding change is due to the brightness of the display, irrespective of its duration.

The following test attempts to discriminate between the two hypotheses. Ss viewed the displays with one eye through filters ranging in optical density from 0 to 2.5 -a range of $316: 1$ in light intensity. The other eye was covered with a patch. Display durations were manipulated to yield equivalent brightness levels, i.e., constant products of display duration and fractional transmission were produced. The time hypothesis predicts that performance is dependent upon display duration, for constant equivalent brightness levels. The brightness hypothesis predicts that performance is dependent upon equivalent brightness, for constant durations. Since the early portion of the IFI function appeared to be the most labile, tests were conducted only at 4-msec and at 64-msec IFI.

The results are summarized in Fig. 11 for displacement tests and in Fig. 12 for the stationarity tests. The

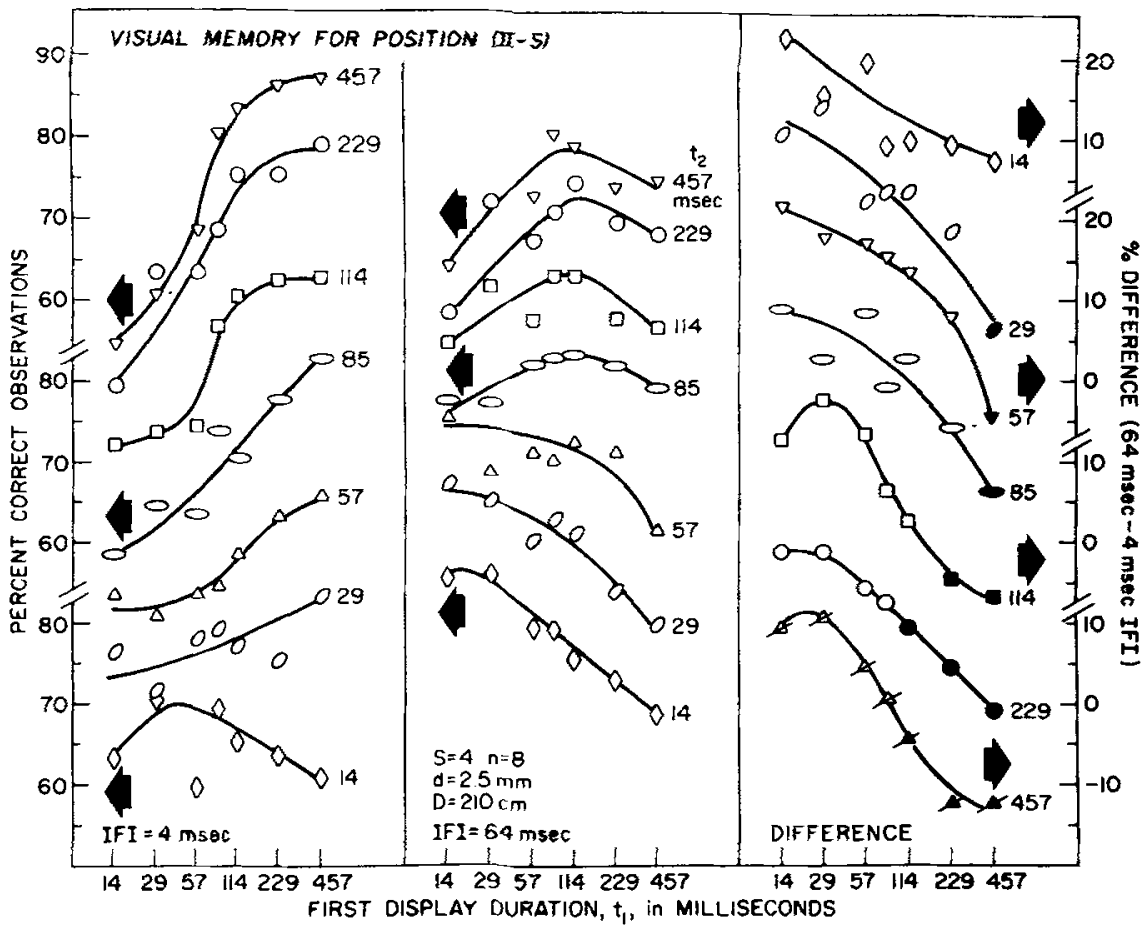

Fig. 14. Stationarity detection under the conditions of Fig. 13. 


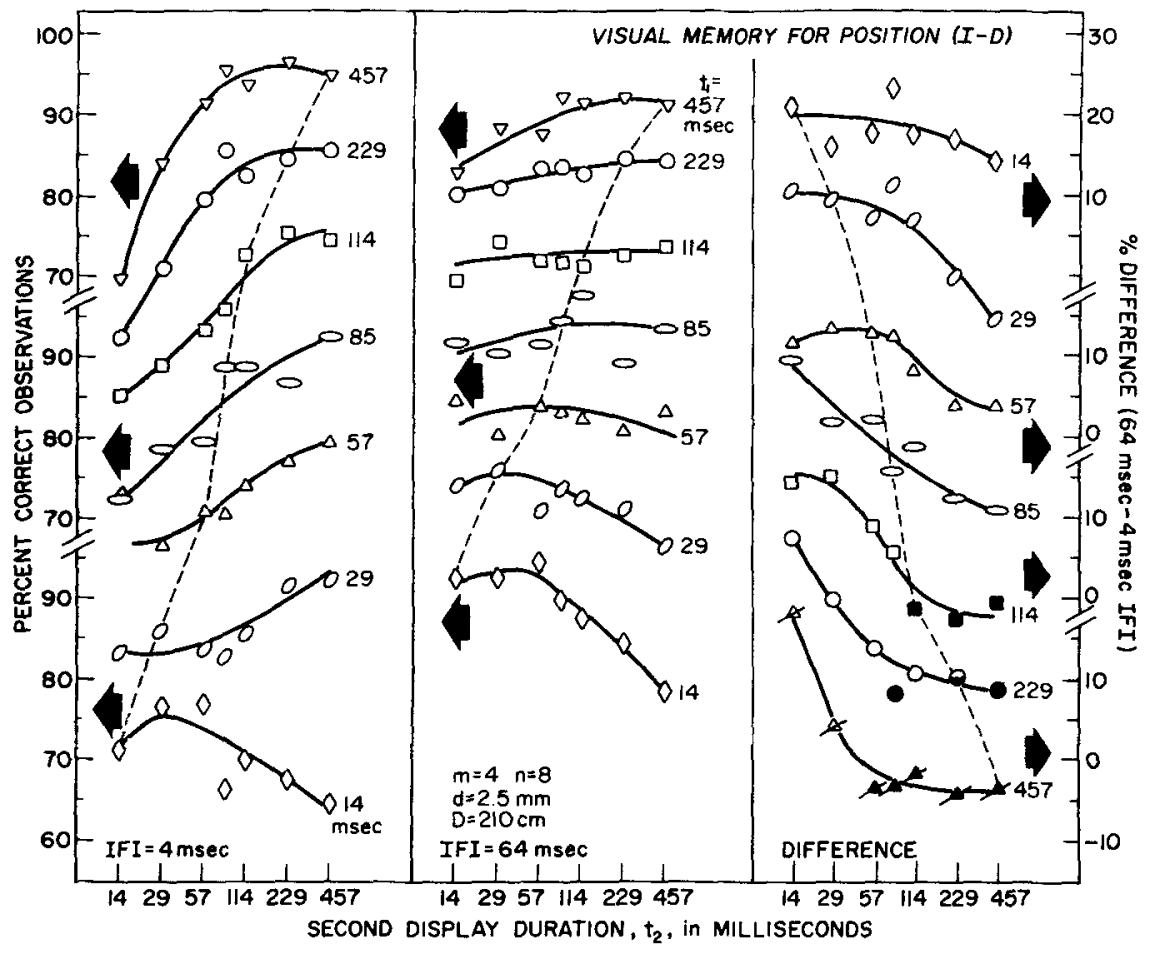

Metacontrast Predictions

It may be that display duration interacts with IFI in a manner parallel to that experienced in metacontrast (Alpern, 1953), although optimal metacontrast and optimal motion is expected for a constant stimulus-onset asynchrony (Kahneman, 1967). Additional tests were carried out in which the duration of the first and second displays could be manipulated independently. While quantitative predictions based on metacontrast are difficult for me to generate, it seemed reasonable to search for strong asymmetries in favor of the second display duration.

The results of these tests are

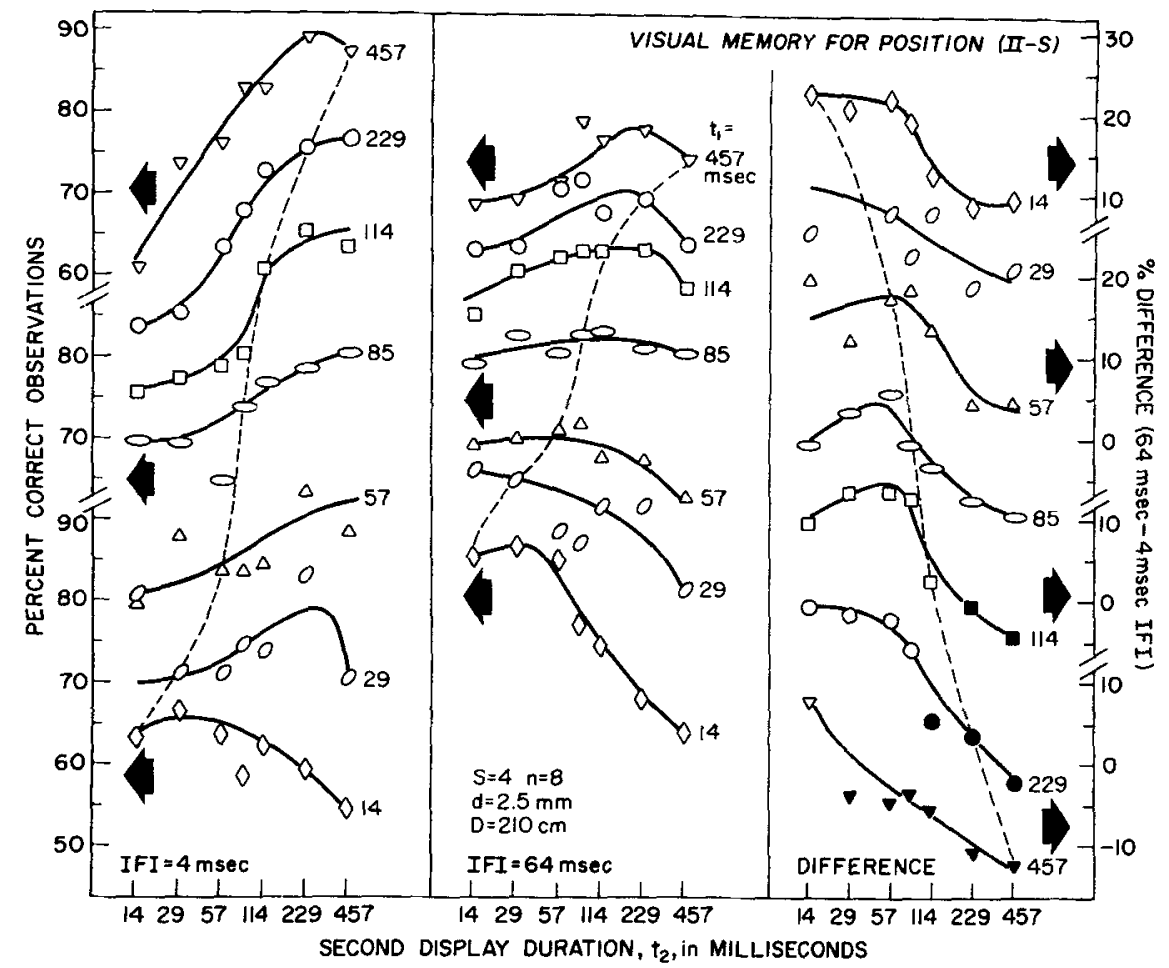

Fig. 15. Displacement detection under the conditions of Fig. 13 with reversal of abscissa and parameter.

considered in Figs. 13-16. The organization of each graph is the same. The two left sections represent performance scores with IFI $=4 \mathrm{msec}$ and IFI $=64 \mathrm{msec}$, respectively; the right section represents the difference scores. In Figs. 13 and 14, the abscissa is the duration of the first display, $t_{1}$, and the parameter is the duration of the second display, $t_{2}$. The same data are plotted in Figs. 15 and 16 with an interchange of the abscissa and parameter. The dashed lines connect points of equal duration for the first and second displays. The detection of displacement is considered in Figs. 13 and 15; the detection of stationarity is considered in Figs. 14 and 16.

While individual functions may differ for given values of $t_{1}$ and $t_{2}$, e.g., $t_{2}=14 \mathrm{msec}$ in the left section of Fig. 13 vs $t_{1}=14 \mathrm{msec}$ in the left section of Fig. 15, the overall impression gleaned is the remarkable near-interchangeability of $t_{1}$ and $t_{2}$. I interpret this result as a rejection of a metacontrast interpretation of the duration-IFI interaction.

\section{Other Hypotheses}

Whereas eye movement requirements suggest that IFIs of $150 \mathrm{msec}$ might be crucial, small shifts in the orientation of the central visual field may be possible at still shorter durations. I know of no evidence to support this proposition.

My initial hypothesis was that IFI was effective for brief, but not for long displays, because the visual image of the first display had to "stabilize" or "settle down" before the second flash could be compared accurately with the first. In this formulation, the duration of the first display is critical, but the duration of the second is not. The results of Figs. 13-16 clearly reject this hypothesis.

Finally, all we may have is the beginning of evidence which calls for an updating and extension of Korte's laws (1915) for "good" visual movement. Since the IFI functions are relatively unchanged by the displacement magnitude, except at extreme values, and since the optimal IFI is independent of both displacement magnitude and of stimulus duration, the present results seem to be in direct contradiction with predictions from Korte's laws.

Finally, a comment is in order with respect to the large differences between the present findings and those

Fig. 16. Stationarity detection under the conditions of Fig. 14 with reversal of abscissa and parameter. 
obtained by Phillips and Baddeley (1971), who obtained significant discrimination (about $65 \%$ correct) for interflash intervals of 3 sec. Their employment of an interflash noise field probably served to disrupt perceived motion. Their employment of a matrix exposed for a long duration may have permitted a crude form of name encoding, or the addition or subtraction of a display element may have been a crude form of number discrimination.

\section{SUMMARY}

I set out to examine the visual memory for spatial position because it seemed to offer a method of examining visual memory for relatively uncoded materials. Along the way, I became fascinated with extremely short-term effects, which must, in some way, be related to basic visual functions. To date, however, simple hypotheses do not fare well in accounting for these effects. I unabashedly solicit help in this regard.

\section{REFERENCES}

ALPERN, M. Metacontrast. Journal of the Optical Society of America, 1953, 43, 648-657.

KAHNEMAN, D. An onset-onset law for one case of apparent motion and metacontrast. Perception Psychophysics, 1967, 2, 577-584.

KINCHLA, R. A. Visual movement perception: A comparison of absolute and relative movement discrimination. and relative movement discrimination. 165-171.

KORTE. A. Kinematoskopische Untersuchungen, Zeitschrift für Psychologie, 1915, 72, 193-206.

LETTVIN, J, Y., MATURANA, Ho R. McCULLOCH, "W. S.. \& PITTS, $\mathbf{W}_{\circ}$ H. What the frog's eye tells the frog's brain. Proceedings of the Institute of Radio Engineers, 1959, 47, 1940-1951.

NEISSER, U. Cognitive psychology. New York: Appleton-Century-Crafts, 1967.
PHILLIPS, W. Ac, BADDELEY, A. D. Reaction time and short-term visual memory. Psy chonomic Science, 1971, 22 , 73-74

POLLACK, I. Message uncertainty and message reception. Journal of the Acoustical Society of America, 1959, 31. 1500-1508

POSNER, M. I., BOIES, S. J., EICHELMAN, W. H., \& TAYLOR, R. L. Retention of visual and name codes of single letters. Journal of Experimental Psychology, 1969, 79, No. 1, Part 2.

POSNER, M. I., \& KEELE, S. W. Decay of visual information from a single letter. Science, 1967, 158, 137-139.

POSNER, M. I \& MITCELL, R. P. Chronometric analysis of classification. Psychological Review, 1967, 74, 392-409.

SEKULER, R. W. A ABRAMS M. Visual sameness: A choice time analysis of pattern recognition processes. Journal of
Experimental Psychology, 1968. 77, 232-238.

SPERLING, G. The information available in brief visual presentations. Psychological Monographs, 1960, 74(11, Whole No. 498).

(Received for publication March 12, 1971.) 